\title{
Névrose obsessionnelle, névrose idéale 1
}

\author{
- Alain Vanier
}

Pour reprendre ici, d'une autre façon, ce que j'ai déjà approché dans un travail précédent ${ }^{2}$, je vais commencer par quelque chose qui m'embarrasse : Je vais faire le portrait de la névrose obsessionnelle, c'est-à-dire proposer une sorte de fiction clinique qui contredit la radicale singularité de notre pratique. Portrait du névrosé obsessionnel en jeune homme, puisque cette névrose a une affinité avec le masculin. L'homme n'a pas le privilège de la névrose obsessionnelle, mais il a une préférence, disait Lacan, pour cette façon de témoigner de l'inaptitude au rapport sexuel. Parler de la névrose obsessionnelle nous fait toujours courir le risque de l'aborder avec ses propres moyens. L'obsessionnel est un penseur, il aime la pensée, la théorie - theoria - le spéculatif, mot particulièrement judicieux dans ce cas.

Je voudrais insister avec un petit appel à la prudence. Notre tâche nécessaire et incessante pourrait s'appuyer sur une question : Comment repenser la clinique ou, plutôt, comment repenser la question de la clinique ? À ce propos, je mets en exergue deux petites interventions de Lacan : «Les sujets d'un type sont donc sans utilité pour les autres du même type. Et il est concevable qu'un obsessionnel ne puisse donner le moindre sens au discours d'un autre obsessionnel. C'est même de là que partent les guerres de religion : S'il est vrai que pour la religion (car c'est le seul trait dont elles font classe, au reste insuffisant), il y a de l'obsession dans le coup. » Quelque temps après, il reprend : «Si j'ai parlé des types cliniques, ce n'est pas sans raison. Je voudrais faire une remarque, c'est que les

1. Texte d'une communication aux Journées d'Études d'Espace analytique, Traiter la névrose des 2 et 3 avril 2005 à Paris.

2. A. Vanier, «Aujourd'hui, la névrose obsessionnelle », L'Évolution psychiatrique $\mathrm{n}^{\circ} 70$, 2005. 
sujets d'un type, hystérique ou obsessionnel, selon la vieille clinique, sont sans utilité pour les autres du même type. Il est plus que concevable, il est touchable du doigt tous les jours qu'un obsessionnel ne puisse donner le moindre sens au discours d'un autre obsessionnel. » Retenons le terme de vieille clinique, celle de laquelle nous procédons, mais aussi celle de laquelle il serait conséquent d'essayer de se séparer, pour réarticuler un peu ce que nous apprend notre pratique. Il manque sans doute encore un pas supplémentaire, ou plutôt simplement de ne pas reculer puisque l'on voit aujourd'hui des cures s'organiser suivant le schéma médical « diagnostic - conduite à tenir ». Pourtant, Freud a apporté sa contribution à la vieille clinique, précisément avec la névrose obsessionnelle. La folie du doute est devenue une névrose et le dégagement de ce qu'il a nommé Zwangsneurose.

Tout d'abord, qu'est-ce qu'une névrose ? Le névrosé est aux prises avec un problème, l'Autre. Qu'est-ce que l'Autre et la nature de ce lien ? Il y a l'Autre du signifiant, l'Autre du langage, et puis il y a l'Autre du corps. Le problème de cette relation avec l'Autre, c'est que l'Autre n'existe pas. Versant signifiant, il est le lieu du signifiant, du trésor des signifiants. Il est l'Autre du savoir et une instance de vérité. Mais notre culture disjoint précisément savoir et vérité, condition paradoxale de la psychanalyse même. Le transfert montre que ce savoir est un savoir supposé. Or, non seulement le névrosé suppose ce savoir chez l'Autre, mais de plus il pense que, de ce savoir, l'Autre jouit. Cette supposition est le propre de la névrose. Le transfert, tel que la psychanalyse ne fait que l'isoler pour le traiter, est la preuve même que l'Autre n'existe pas. Le propre du névrosé est de supposer du sujet chez l'Autre et, ainsi, dans cette configuration, c'est l'Autre qui demande, l'Autre qui désire. Mais cet Autre qui n'existe pas, il revient au sujet de le faire exister. Le transfert, au sens le plus large du terme, constitue la dépense du névrosé pour faire exister l'Autre, le lieu de son sacrifice permanent. Ça peut s'organiser collectivement, les religions, les grandes causes; ou se manifester plus individuellement. Pour le faire exister, il vire ce reste non symbolisable, que Lacan a nommé objet a, au compte de l'Autre, et il fait ainsi de cet objet la cause de son désir, avec quoi il construit son fantasme. Cet objet paradoxal a été laissé en gage à l'Autre pour s'en séparer, tout autant que pour ne pas risquer d'être cet objet. Mais, en même temps, les névrosés sont des mal séparés. Ainsi, le désir qui est le propre du névrosé, ce désir est une défense contre la jouissance, une défense contre le réel. Il ne vise pas un objet dont il pourrait jouir ; bien au contraire, la stratégie du névrosé, c'est de tenter de résoudre ce désir en demande : soit il demande cet objet supposé chez l'Autre, soit il pense qu'on lui 
demande de régler la dette ou le prix d'une jouissance. «Le névrosé, c'est quelqu'un qui n'arrive pas à ce qui pour lui est le mirage où il trouverait à se satisfaire, à savoir une perversion : une névrose, c'est une perversion ratée. " Cette jouissance, manière de la tenir à distance, est bordée par l'angoisse. C'est pourquoi il est anxieux. Car de l'Autre du corps, d'un autre corps, nous dit Lacan, « on ne peut rien en faire, à part le mettre en morceaux " .

Et pourtant, il a joui ! Le problème de l'obsessionnel est qu'il ne parvient pas à l'oublier. Il connaît ses traumas, souligne Freud, mais ignore leur valeur. Il fabrique de la culpabilité avec son angoisse. Là où l'hystérique parvient à élaborer un compromis entre des tendances contradictoires et construit un symptôme qui permet une satisfaction érotique, une jouissance, l'obsessionnel l'élude, le contourne : ces tendances contradictoires sont exprimées successivement, d'où le doute, les actes compulsifs qui produisent la montée d'une tension, d'une jouissance et non sa résolution.

Dans la névrose obsessionnelle, deux pulsions intéressent ces morceaux du corps que sont les formes particulières de l'objet a : la pulsion de regarder et de savoir, indique Freud, et la pulsion anale, deux pulsions qui mettent en jeu un objet externe.

Dans un premier temps, l'enfant, dans son rapport à l'objet oral, croit que l'objet partiel, que le sein, c'est l'Autre, il ne sait pas qu'il est cet être plaqué sur la mamelle. C'est seulement au niveau anal, pour la première fois, qu'il peut se reconnaître dans un objet, car l'objet anal est subjectivé par la demande de l'Autre. Et cette demande de la mère - c'est la difficulté de l'obsessionnel - commande à la fois de retenir et de donner. L'objet prend ainsi presque la valeur d'une partie du corps, puisqu'il est à l'intérieur. Mais la demande, comme don, est ambiguë car cet objet regardé, admiré est aussitôt jeté ${ }^{3}$. Cette reconnaissance particulière peut déjà suffire à expliquer le doute, l'hésitation, l'incertitude constante du sujet. Néanmoins, comme tout névrosé, il a affaire à l' đEdipe. Mais le passage au niveau phallique ne va pas réduire cette position, car le lien à l'objet anal va lui permettre de contourner le phallus. Cet objet - fèces, excréments symbolise, incarne merveilleusement le phallus : il est là, il n'est pas là, il apparaît puis disparaît. Confronté à l'impossibilité de se satisfaire au niveau phallique, l'objet anal vient faire bouchon, il image la perte du phallus. On saisit ainsi la

3. Cf. J. Lacan (1962-1963), L'angoisse. Le Séminaire. Livre X, texte établi par J.-A. Miller, Paris, Le Seuil, 2004. 
raison des affinités masculines de cette structure. Lacan relève, à ce propos, que la fantaisie de la relation génitale comme don, est obsessionnelle.

Le niveau anal vient recouvrir le réel du non rapport, et l'objet anal retient le sujet sur le bord du «trou castratif », mieux même, il représente le sujet. Ainsi, il construit l'idée d'un don dans l'acte génital. « Il engage l'excrément de lui dans l'amour. » L'envers de la merde, c'est l'idéalisation. C'est la double polarité de la valeur de cet objet. Au niveau de la demande, le lien anal paraît tout symboliser. Il reste le désir, nécessaire pour ne pas se réduire à l'objet de la demande, ce qui advient quand il se déprime. Il ne se prend pas pour de la merde, dit-on souvent de lui : mais si, justement, ce qu'il redoute est d'être cet objet qui doit le séparer. Là s'articule le niveau scopique. L'acte anal implique une maîtrise motrice, or le support narcissique de la maîtrise de soi est spéculaire, support où se détache l'objet, d'autant plus qu'il émane d'une partie du corps que le sujet ne voit pas. Le niveau scopique, souligne Lacan, est celui qui masque le mieux la castration. L'idéalisation est bien l'autre versant, le corrélat du premier niveau pulsionnel. Cet idéal se figure comme dieu, en tant qu' omnivoyant », œil qui contrôle, observe toutes nos actions. D'où les dédoublements du névrosé, déjà relevés par Freud, par exemple entre la femme idéalisée, qu'on ne touche pas ou plus, et la femme ravalée, ou bien entre lui et lui-même, entre l'image qu'il promène et présente en la détachant littéralement de son corps. La névrose obsessionnelle est le principe de la conscience, mais c'est une conscience au spectacle, qui se dédouble entre la position de l'acteur et celle du spectateur, duplicité qui rend compte de ses constantes oscillations. Ces mouvements se manifestent en permanence, par exemple dans l'alternance surprenante entre des moments d'avarice extrême et des moments de prodigalité excessive. Il se retient, mais, à d'autres moments, il ne peut pas se retenir, il est empêché de se retenir, d'où les compulsions. En fait, il cherche l'élément initial, la cause première qui permettrait de boucler enfin ce circuit toujours ouvert, mais cet objet ultime est dérisoire. Il le cherche, nous dit Lacan, mais il ne faut pas le trouver. C'est le chercheur parfait - la recherche qui cherche -, il cherche infiniment pour ne pas trouver. Il tourne en rond puisqu'il détruit tous les objets de substitution, à mesure qu'il s'en approche. À mesure qu'il s'approche de son désir, il est saisi par le doute, et l'objet lui-même est désinvesti. Mais qu'il perde cet objet indifférent, que la femme délaissée le quitte, aussitôt cet objet perdu est réinvesti.

L'obsessionnel va recouvrir le désir par la demande, et sa stratégie dans la cure consiste à faire surgir la demande de l'Autre. C'est une indication pour nous dans ce traitement : il suffit que vous parliez, c'est une demande et ça peut suffire ; 
d'ailleurs, même si vous ne dites rien... C'est un des problèmes constants des cures d'obsessionnels.

L'objet cause anal est donc recouvert par un mirage spéculaire au titre d'une puissance qui est virée au compte de l'Autre. Or, sur le versant scopique, ce qui est en jeu n'est pas tellement le niveau du désir de l'Autre, qui intéresse plutôt la voix, mais plutôt celui du « désir à l'Autre », comme point d'aspiration à la fois quêté et redouté. Il témoigne de la puissance de captation de l'image du corps. Il va s'agir de construire cet Autre dans le regard, l'Autre qu'il faut faire jouir. On a pu dire que l'obsessionnel est comme la grenouille qui veut se faire aussi grosse que le bœuf. En effet, sous le regard de cet Autre, l'obsessionnel va accomplir les exploits par lesquels il rivalise avec le phallus imaginaire. Mais il lui faut inlassablement recommencer, parce qu'il est inévitablement dépossédé de la jouissance attendue, qui est aussitôt virée au compte de l'Autre. L'un de mes patients, alpiniste, répondait invariablement à qui l'interrogeait sur les dangers de cette pratique : «Il suffit de ne pas faire de faute. »

Ce phallus imaginaire n'est pas le père. Quelque chose a dérapé au niveau de la métaphore paternelle : il y a une certaine carence du père qui accentue la dimension imaginaire. Le père est moins une cause qu'un alibi, qui se présente au centre de la névrose obsessionnelle. Le dernier livre de Markos Zafiropoulos montre le point d'émergence du Nom-du-Père chez Lacan comme ce qui particularise une certaine culture - la nôtre ${ }^{4}$. Il faut tuer le père. C'est une formule obsessionnelle qui sous-entend qu'il est toujours vivant. C'est pourquoi Lacan situe Totem et tabou comme un mythe obsessionnel. La proximité du père et de la jouissance est le problème de l'obsessionnel. Il a un père qui jouit mais qui a failli au niveau de son désir. Ce père que l'obsessionnel doit maintenir vivant en permanence est un père qui parle, qui jouit, et qui nourrit son interrogation perpétuelle sur son désir et sa jouissance. Or, précisément, l'obsessionnel voudrait réduire le père au signifiant, en faire un père symbolique, parce qu'il y a pour lui un défaut de garantie de la loi, il a constamment peur qu'elle défaille. D'où le recours si fréquent à la religion, et sa paradoxale panique quand on lui annonce la fin du père, qui n'est peut-être pas sans rapport avec celle qui saisit certains de nos collègues à l'annonce de son déclin.

L'obsessionnel passe son temps à faire les comptes de son rapport à l'Autre, mais ceux-ci « ne tombent jamais justes », d'autant que, si l'Autre n'est pas mort,

4. M. Zafiropoulos, Lacan et Lévi-Strauss, Paris, PUF, 2003. 
il peut réclamer ce qui lui est dû pour la jouissance. Il est dans la quête terrifiante de ce qui reste de la Chose, toujours à proximité, et qui alimente, dans le même temps, sa crainte. On peut penser ici à la compulsion de l'Homme aux loups à voir les femmes nues. Il cherche ainsi à s'assurer l'Autre parce que l'Autre est trop réel. Il ne se prend pas pour un maître, ce qui ne veut pas dire qu'on ne le prenne pas pour tel. Le fondement du couple répandu de l'obsessionnel et de l'hystérique peut se comprendre ainsi : l'hystérique cherche un maître. Quant à lui, il rencontre chez l'hystérique cette jouissance énigmatique qui le fascine et reste à maîtriser. L'illusion de maîtrise pourtant s'épuise rapidement, et l'empêchement obsessionnel figurera assez bien sa castration, donnant au couple une nouvelle assiette. Quant à lui, il règlera l'angoisse de son désir en le rabattant sur la demande : ce qu'elle veut, c'est le phallus. Ce qui parasite sa relation aux autres tient justement à cette question : que veut-il ? Il peut la transformer en demande : que me veut-il ?, ou fomenter la figure d'un maître qui sache ce qu'il veut, c'est-à-dire un Père idéal. Freud a mis un certain temps pour distinguer le surmoi et l'idéal du moi, le versant de l'idéal, et celui du surmoi comme appel à la jouissance pure. Angoisse devant le surmoi, car c'est le père qui dit « Jouis ! » ce qui est « impossible à satisfaire aux termes de la conscience morale ». Une jouissance, demandée et commandée, se manifeste dans ces oscillations. Pour ne pas se perdre, il doit s'empêcher de disparaître, de s'effacer comme sujet et, donc, ne pas se soumettre à la division subjective pour ne pas risquer d'être l'objet. Cet accent se traduit par ce gonflement du moi toujours cliniquement remarquable.

Le discours a pour fonction de recouvrir le non-rapport. Ainsi, nous tissons des liens sociaux, et s'organisent des agencements collectifs. Si l'on considère le discours de l'hystérique, nécessaire dans l'engagement d'une analyse, l'obsessionnel rencontre une difficulté avec la place de l'autre du discours, lié à l'ambivalence entre destruction et soutien de cette place. Mais comme variante de l'hystérie, dialecte, une voie est possible qui passe par une certaine hystérisation, pour éviter l'écueil du discours du maître qui peut fonctionner comme impasse, tout en organisant du lien social et en permettant une certaine stabilisation. Je rejoindrai les conclusions du travail antérieur que j'ai évoqué en commençant par vous lire deux propositions de Lacan, et, tout d'abord : « Les névroses, ça existe. Je veux dire qu'il n'est pas très sûr que la névrose hystérique existe toujours, mais il y a sûrement une névrose qui existe, c'est ce qu'on appelle la névrose obsessionnelle. » Auparavant, il affirmait : « L'homme, on le sait d'expérience, n'a pas le privilège de la névrose obsessionnelle, mais il a une préférence pour cette façon de témoigner de l'inaptitude au rapport sexuel qui n'est pas le lot de son sexe. 
Ce témoignage n'a pas moindre valeur que le témoignage de l'hystérique. Il a pourtant moins d'avenir, non pas seulement d'avoir un passé très chargé, mais de ne trouver place dans aucun discours qui tienne. " Ainsi, l'hystérie se mue en discours, la névrose obsessionnelle reste la névrose de cette fin de siècle, sans doute du fait de son asocialité, de sa pente à l'isolement en ces temps de loneliness, comme a pu le dire Arendt, mais aussi parce qu'il témoigne mieux qu'un autre de l'imminence de la jouissance, mieux voilée par l'impuissance du père qu'agence discursivement l'hystérie. Que le plaisir soit une barrière à la jouissance, c'est ce qu'on observe tous les jours. Dans notre civilisation des objets, le plaisir devient la limite majeure du parasitage qui menace tout un chacun. L'obsessionnel incarne assez bien cette figure de la modernité consommatrice, de cette jouissance solitaire que permettent les avancées techniques, chacun comblé par les objets d'un voyeurisme généralisé, gadgets où le sujet puise une satisfaction paradoxale pour un Autre machinisé, celui de tous ces objets dont Hannah Arendt notait qu'ils n'étaient produits que pour être détruits, et l'on peut se demander si cette perspective de destruction n'est pas ce qui leur confère une certaine valeur. Quant à l'idéal de transparence et de maîtrise, il explique sans doute l'engouement pour les thérapies comportementales et leurs dérivés.

Il y a une modernité de la névrose obsessionnelle, car son émergence semble liée à cet affaiblissement de la fonction du père, à cet apparent déclin du religieux avec un Dieu qui ne prend plus aussi efficacement sur lui nos fautes et dont le retour est à chaque fois plus terrible. De l'hystérie, Lacan a fait un discours, un lien social qui trouve une certaine assiette dans son rapport aux nouvelles figures de maîtrise. La névrose obsessionnelle isole. Elle s'isole sous la domination des petits leaders, là où l'hystérique déplore chaque jour un peu plus la disparition d'un vrai. Néanmoins, l'obsession se satisfait aussi de leur fragilité, ce qui lui donne maintenant une certaine mobilité. La faillite des pères et le problème contemporain du religieux ne nous montre-t-il pas cet obsessionnel si avare comme le grand dépensier qui soutient ainsi la structure ? Si le soubassement de toute névrose est hystérique car lié au non rapport sexuel, l'hystérique le reconstitue, le recouvre par un discours, là où l'obsessionnel en présente une autre face, un autre dialecte, dont témoigne le repérage théorique devenu possible du père comme symptôme, dans notre civilisation « fortement centrée sur ses névrosés ». Sans doute pour cela, dans ce monde où l'on se mêle de plus en plus, et qui rend la jouissance de l'Autre plus proche, trop proche, Lacan pouvait annoncer la montée des ségrégations, le retour du religieux, non pas comme équivalent strict de la névrose obsessionnelle, mais qui, comme elle, et avec toute la polysémie du 
terme constitue la névrose idéale ; idéale pour témoigner de notre époque comme l'hystérie, une certaine hystérie, le fut, il y a plus d'un siècle.

\section{Résumé}

Tableau de la névrose obsessionnelle sur le versant du statut de l'objet et de la jouissance. Sous cet angle, la névrose obsessionnelle pourrait bien apparaître comme la névrose « idéale » de notre époque.

\section{MOTS-CLÉS}

Névrose obsessionnelle, névrose idéale

\section{SUMmaRY}

Brief description of obsessional neurosis with emphasis on the status of the object and jouissance. Seen from this angle, obsessional neurosis might well be considered as the «ideal » neurosis for our times.

\section{KEY-WORDS}

Obsessional Neurosis, Ideal Neurosis. 